\title{
Helical phase inflation via non-geometric flux compactifications: from natural to starobinsky-like inflation
}

\author{
Tianjun $\mathbf{L i}^{,}{ }^{a, b}$ Zhijin $\mathbf{L i}^{c}$ and Dimitri V. Nanopoulos ${ }^{c, d, e}$ \\ ${ }^{a}$ State Key Laboratory of Theoretical Physics and \\ Kavli Institute for Theoretical Physics China (KITPC), Institute of Theoretical Physics, \\ Chinese Academy of Sciences, Beijing 100190, P.R. China \\ ${ }^{b}$ School of Physical Electronics, University of Electronic Science and Technology of China, \\ Chengdu 610054, P.R. China \\ ${ }^{c}$ George P. and Cynthia W. Mitchell Institute for Fundamental Physics and Astronomy, \\ Texas A\&M University, College Station, TX 7r843, U.S.A. \\ ${ }^{d}$ Astroparticle Physics Group, Houston Advanced Research Center (HARC), \\ Mitchell Campus, Woodlands, TX 77381, U.S.A. \\ ${ }^{e}$ Academy of Athens, Division of Natural Sciences, \\ Athens 10679, Greece \\ E-mail: tli@itp.ac.cn, lizhijin@physics.tamu.edu, \\ dimitri@physics.tamu.edu
}

ABstract: We show that a new class of helical phase inflation models can be simply realized in minimal supergravity, wherein the inflaton is the phase component of a complex field and its potential admits a deformed helicoid structure. We find a new unique complex-valued index $\chi$ that characterizes almost the entire region of the $n_{s}-r$ plane favored by new Planck observations. Continuously varying the index $\chi$, predictions interpolate from quadratic/natural inflation parameterized by a phase/axion decay constant to Starobinsky-like inflation parameterized by the $\alpha$-parameter. We demonstrate that the simple supergravity construction realizing Starobinsky-like inflation can be obtained from a more microscopic model by integrating out heavy fields, and that the flat phase direction for slow-roll inflation is protected by a mildly broken global U(1) symmetry. We study the geometrical origin of the index $\chi$, and find that it corresponds to a linear constraint relating Kähler moduli. We argue that such a linear constraint is a natural result of moduli stabilization in Type II orientifold compactifications on Calabi-Yau threefolds with geometric and non-geometric fluxes. Possible choices for the index $\chi$ are discrete points on the complex plane that relate to the distribution of supersymmetric Minkowski vacua on 
moduli space. More precise observations of the inflationary epoch in the future may provide a better estimation of the index $\chi$. Since $\chi$ is determined by the fluxes and vacuum expectation values of complex structure moduli, such observations would characterize the geometry of the internal space as well.

KeYwords: Supergravity Models, Flux compactifications, Supersymmetric Effective Theories

ARXIV EPRINT: 1507.04687 


\section{Contents}

1 Introduction 1

2 Starobinsky-like helical phase inflation $\quad 4$

3 Global U(1) symmetry in the superpotential $\quad 8$

4 Moduli constraint from flux compactification $\quad 11$

$\begin{array}{lll}4.1 & \text { Type IIA compactification with geometric fluxes } & 11\end{array}$

$\begin{array}{lll}4.2 & \text { Type IIB compactification with Q-fluxes } & 13\end{array}$

$\begin{array}{llr}5 & \text { Conclusions } & 18\end{array}$

\section{Introduction}

Inflation [1-7] has attracted widespread attention in the past few decades. The inflationary epoch is crucial for the cosmic evolution, and provides a unique opportunity to probe physics close to the grand unification scale, far beyond the scope directly accessible in the laboratory. A principle challenge to the construction of inflationary models within the $N=1$ supergravity or superstring theories is the so-called $\eta$ problem. Specifically, the inflaton potential obtained in these contexts is usually too steep to trigger slow-roll inflation. Moreover, it requires trans-Planckian field excursion for the generation of sizable tensor fluctuations [8], rendering Planck-suppressed higher-dimensional operators non-negligible.

Helical phase inflation $[9,10]$ was proposed as a solution to both the $\eta$ problem $^{1}$ and the trans-Planckian field excursion problem. In helical phase inflation the inflaton is a pseudoNambu-Goldstone boson (PNGB), the phase component of a complex field. A PNGB was first employed as the inflaton in $[22,23]$ in order to protect the flat potential against quantum loop corrections. The potential of a complex field admits helicoid structure, and during inflation its radial component is strongly stabilized, while the inflaton evolves along a local valley, tracing a beautiful helical trajectory. In this model, the $\eta$ problem is automatically solved by the global U(1) symmetry of the minimal Kähler potential $K=$ $\Phi \bar{\Phi}$. This U(1) symmetry is broken in the holomorphic superpotential and leads to phase monodromy. Phase rotation provides a proxy for trans-Planckian field excursion, whereas the "physical" field does not evolve into the super-Planckian domain, where quantum gravity effects are likely to break slow-roll conditions. As argued in [10], such supergravity constructions are necessarily effective descriptions of a more fundamental theory with heavy fields integrated out.

\footnotetext{
${ }^{1}$ The $\eta$ problem can also be solved by Heisenberg symmetry in no-scale supergravity [11-17] or shift symmetry in minimal supergravity [18]. Supergravity inflation with broken shift or global U(1) symmetries was studied in [19-21].
} 
In helical phase inflation, the helical trajectory and phase monodromy of the superpotential are similar to the axion monodromy inflation scenario, as realized via the DBI action of wrapped D5-branes in ref. [24, 25]. Likewise, the axion alignment mechanism was suggested in ref. [26], in order to obtain super-Planckian axion decay constant, and was investigated as a new type of axion monodromy in [27-29]. Inflaton dynamics in helical phase inflation associated with explicit breaking of the global U(1) symmetry may be similarly dubbed phase-axion alignment. Additionally, a PNGB has been employed as the inflaton in recent studies on inflation models with stabilized or almost stabilized radial component [21, 30-38]. However, most of these models do require super-Planckian field excursion during inflation and predict large tensor fluctuation with a tensor-to-scalar ratio $r \sim 0.1 .^{2}$ Recent Planck observations on cosmic microwave background [40, 41], as well as a joint analysis utilizing B-mode polarization data from the BICEP2/Keck Array [42], have provided tighter constraints on inflationary observables, particularly the tensor-to-scalar ratio, which is $r<0.08$ at the $95 \%$ confidence level. As a consequence, large field inflation models with power-law potentials $V(\phi) \propto \phi^{n}$ have been ruled out for $n \geqslant 2$, and natural inflation is now also in tension with current data. In contrast, the Starobinsky model [1] predicts a small tensor-to-scalar ratio $r \simeq 0.003$, which remains entirely consistent with the latest observations.

Since the discovery of no-scale supergravity realizations of the Starobinsky model $[43$, 44], numerous generalization and extensions of the idea have been proposed. Specifically, by introducing one additional parameter, the Starobinsky model can interpolate to quadratic or natural inflation [44-54]. The problem of trans-Planckian field excursion has been carefully considered in quadratic and natural inflation, while it usually is ignored for Starobinsky-like inflation, since the tensor-to-scalar ratio is lower than the Lyth bound $r \sim 0.01$. For Starobinsky-like inflation with a potential

$$
V(\phi)=M^{4}\left(1-e^{-\alpha \phi}\right)^{2},
$$

the tensor-to-scalar ratio is given by $r=\frac{8}{\alpha^{2} N^{2}}$, with an e-folding number $N \in[50,60]$. For small $\alpha \leqslant 0.5$, the tensor-to-scalar ratio is above the Lyth bound, and the model approximates quadratic inflation. For larger $\alpha$, the field excursion during inflation can be expressed as

$$
\Delta \phi \approx \frac{1}{\alpha} \log \left(2 \alpha^{2} N\right)
$$

in Planck units $\left(M_{P}=1\right)$, which reduces to $\Delta \phi \approx 5$ for typical parameter values $\alpha=\sqrt{\frac{2}{3}}$ and $r \approx 0.003$. Therefore, the Starobinsky-like inflation scenario is indeed subject to transPlanckian field excursions, even though the tensor-to-scalar ratio is below the Lyth bound. In order to avoid higher order corrections from quantum gravity effects, which are important in the super-Planckian regime and are likely to violate the slow-roll criteria, related models of inflation must be studied in the context of a UV-completion, such as string theory. Starobinsky-like inflation with a string-theoretic embedding has been studied in refs. [5558]. Another interesting solution is the realization of Starobinsky-like inflation within the

\footnotetext{
${ }^{2}$ It is shown in [39] that small tensor-to-scalar ratio can be generated in aligned natural inflation initiated close to a saddle point.
} 
sub-Planckian region, while allowing trans-Planckian field excursions to be undertaken by an "unphysical" degree of freedom, such as the phase of a complex field which does not admit any polynomial higher order corrections.

In this work, we show that Starobinsky-like inflation can be simply realized based on the supergravity setup for helical phase inflation [10]. Actually, the supergravity setup for Starobinsky-like helical phase inflation is the same as for natural inflation, except that the latter case features a real-valued superpotential parameter that is pure imaginary in the former case. Admitting a complex-valued phase for this parameter, predictions for $n_{s}-r$ thereby interpolate between natural inflation and Starobinsky-like inflation, and regions of the $n_{s}-r$ plane favored by new Planck observations may be characterized by a single complex index $\chi$. The supergravity model is expected to be obtained from a more microscopic model after integrating out heavy fields. In particular, we find that the index $\chi$ has an interesting geometrical origin associated with non-geometric flux compactification.

Non-geometric fluxes are motivated from T-duality between the Type IIA and IIB string theories $[59,60]$. In the low-energy effective $N=1$ supergravity theory obtained from type II string compactification, T-duality is preserved in the action for RR fluxes while this is not the case for NSNS fluxes, leading to the expectation of new fluxes that are T-dual to the NSNS variety. The geometric flux arises from the NSNS flux by invoking T-duality along a direction of the internal space, and it relates to compactification on a twisted torus. By taking T-duality along extra internal directions, one obtains $Q$ or $R$ type fluxes without clear geometric explanation. Geometric and non-geometric fluxes introduce coupling terms in the superpotential for Kähler moduli and uplift these directions at the perturbative level so that they can play important roles in string phenomenology. Moduli stabilization and supersymmetric Minkowski vacua based on non-geometric flux compactification have been studied extensively in refs. [61-69]. It should be noted that, distinct from NSNS and RR fluxes, compactifications with non-geometric fluxes suffer from the dilution problem. After turning on non-geometric fluxes, back-reaction on the internal metric can not be treated by taking a large volume limit with diluted fluxes. The fourdimensional vacua with non-geometric fluxes have been uplifted to ten dimensions based on the $\beta$-supergravity framework [71]. The effective supergravity action from non-geometric fluxes is expected to partially reflect the dynamics around vacua of full string theory.

This paper is organized as follows. In section 2, we study Starobinsky-like helical phase inflation and compare its predictions with new Planck observations, showing that the complex-valued index $\chi$ can characterize regions in the $n_{s}-r$ plane preferred by new Planck data. In section 3 we study a more fundamental realization of helical phase inflation based on both perturbative and non-perturbative effects. The phase monodromy is identified as a global U(1) symmetry mildly breaking at the inflation energy scale. In section 4 we study the geometrical origin of the index $\chi$ in type II orientifold compactifications with geometric and non-geometric fluxes and show that the index $\chi$ is determined by the flux quanta and vacuum expectation values of complex structure moduli. Conclusions are given in section 5 . 


\section{Starobinsky-like helical phase inflation}

The $N=1$ minimal supergravity setup for Starobinsky-like helical phase inflation is rather simple, with the Kähler potential and superpotential given as follows: ${ }^{3}$

$$
K=\Phi \bar{\Phi}+X \bar{X}-g(X \bar{X})^{2}, \quad W=a \frac{X}{\Phi}\left(\Phi^{i c}-1\right) .
$$

A similar supergravity model was proposed in [10] for natural inflation, wherein the superpotential contains a real parameter in the term $\Phi^{b}, b \ll 1$ instead of $\Phi^{i c}$. Here, the imaginary exponent of $\Phi$ seems to be unusual at first glance, although we will show that it has a clear geometrical origin associated with non-geometrical flux compactification of type IIB superstring theory. There is a global U(1) symmetry in the Kähler potential, shifts in which introduces phase monodromy in the superpotential $W$ :

$$
\Phi \rightarrow \Phi e^{2 \pi i}, \quad K \rightarrow K, \quad W \rightarrow W+a \frac{X}{\Phi} \Phi^{i c}\left(e^{-2 \pi c}-1\right) .
$$

By employing the phase of $\Phi$ as an inflaton, the well-known $\eta$ problem for supergravity inflation is absent, since the Kähler potential is phase independent. The phase monodromy in (2.2) never reverts to the original $W$, and so is different from that associated with natural inflation [10], wherein the superpotential is cyclically restored after a sufficient long phase rotation $(\Delta \theta>2 \pi$ with super-Planckian phase/axion decay constant). Similar differences also exist between the respective inflaton potentials.

In $N=1$ supergravity, the F-term scalar potential is determined by the Kähler potential $K$ and the superpotential $W$

$$
V=e^{K}\left(K^{i \bar{j}} D_{i} W D_{\bar{j}} \bar{W}-3 W \bar{W}\right),
$$

where $K_{i \bar{j}}=\partial_{i} \partial_{\bar{j}} K$ and $D_{i} W=\partial_{i} W+K_{i} W$. During inflation the field $X$ is strongly fixed at its vacuum expectation value $\langle X\rangle=0$. The F-term scalar potential then simplifies to

$$
\begin{aligned}
V(r, \theta)=e^{K} D_{X} W D_{\bar{X}} \bar{W} & =a^{2} \frac{e^{r^{2}}}{r^{2}}\left(e^{-2 c \theta}+1-\left(r^{i c}+r^{-i c}\right) e^{-c \theta}\right) \\
& =a^{2} \frac{e^{r^{2}}}{r^{2}}\left(e^{-2 c \theta}+1-2 \cos (c \log r) e^{-c \theta}\right)
\end{aligned}
$$

in Planck units $\left(M_{P}=1\right)$, with $\Phi \equiv r e^{i \theta}$. The $r$-dependent terms in $V, e^{r^{2}} / r^{2}$ and $-2 \cos (c \log r) e^{-c \theta}$ all have minima at $r=1$, irrespective of $\theta$. Therefore, the field norm $|\Phi|$ is strongly stabilized at $\langle|\Phi|\rangle=1$, and the residual phase-dependent potential reduces to

$$
V(\theta)=a^{2}\left(1-e^{-c \theta}\right)^{2},
$$

with the rescaled parameterization $a \rightarrow a \sqrt{e}$. The potential $V(r, \theta)$ is given in figure 1 , for the parameter selection $c=0.6$. The potential shows a deformed helicoid structure. Figure 2 gives the helicoid potential in terms of $r$ and $\theta$ which clearly shows the potential

\footnotetext{
${ }^{3}$ In this model two superfields are employed, recently it was shown in [70] that the helical phase inflation can also be realized with only one superfield.
} 


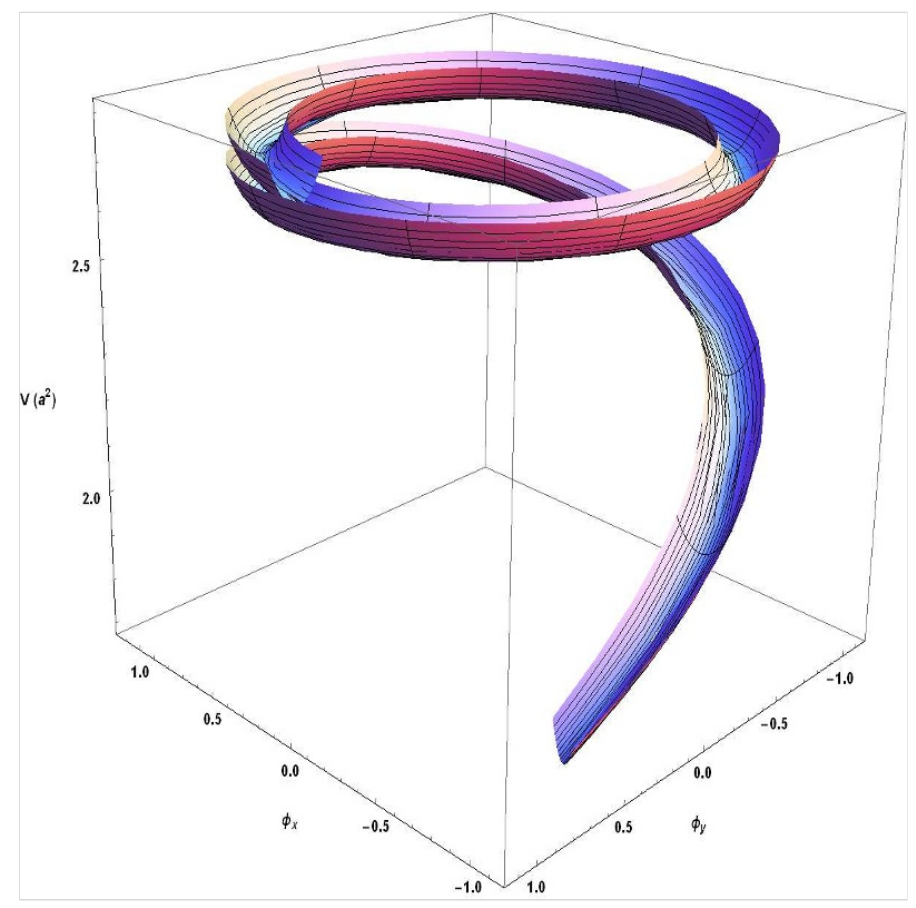

Figure 1. The helicoid potential with unit $a^{2}$ and $c=0.6$. The radial direction has a minimum at $|\Phi|=1$ where the field norm is strongly stabilized during inflation, while the phase direction is sufficiently flat to generate slow-roll inflation. Super-Planckian inflaton excursion is manifest along the phase direction rather than by a physical field.

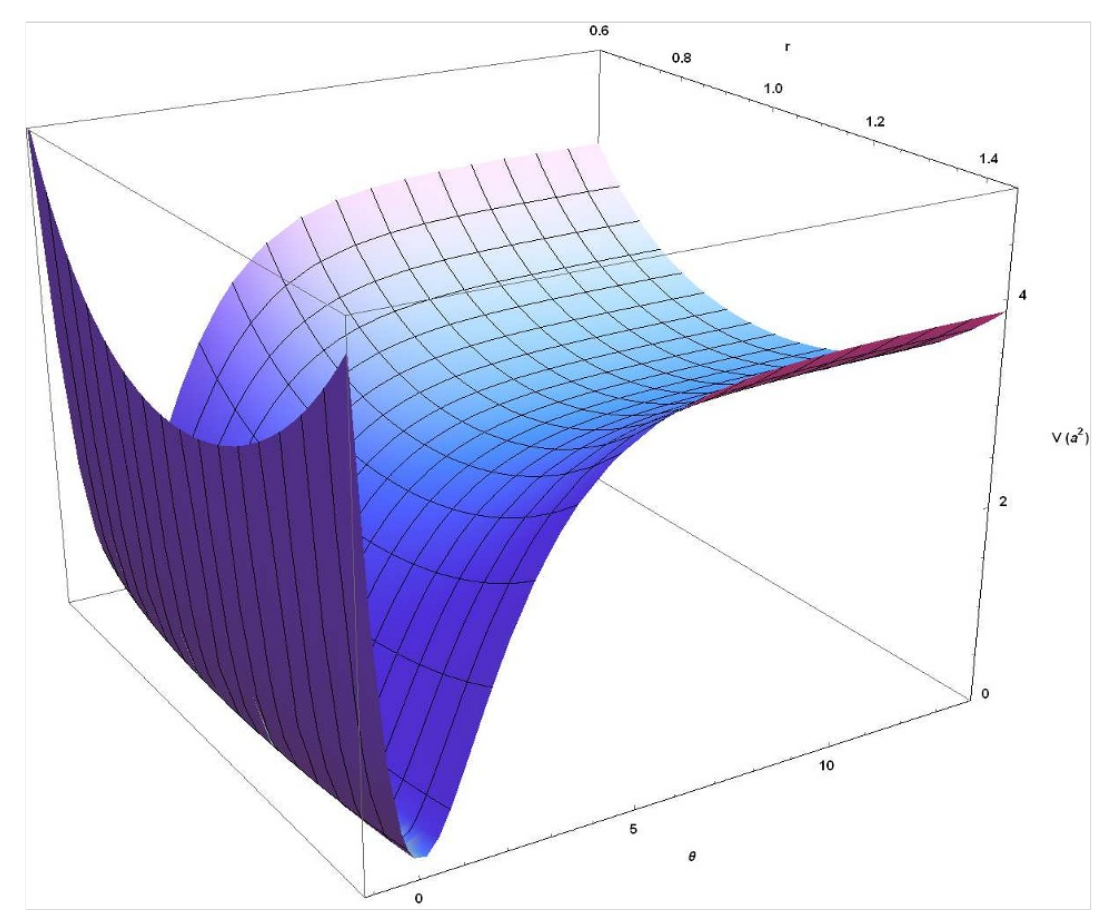

Figure 2. The same potential as in figure 1 in terms of $r \equiv|\Phi|$ and $\theta \equiv \arg (\Phi)$. The local minimum along $r=1$ is clearly shown in the figure. 


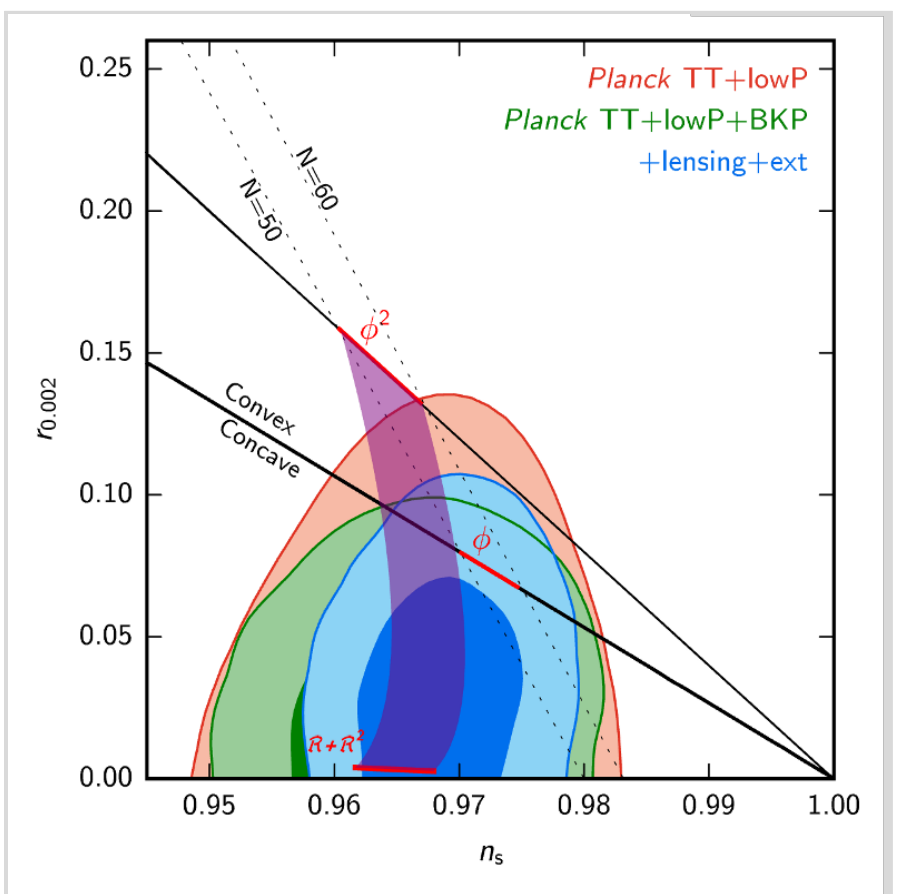

Figure 3. The predictions of Starobinsky-like inflation (purple strip), and new experimental data from ref. [40].

reaches its local minimum at $r=1$. In figure 1 the minimum in the radial direction represents a deformed helical trajectory along which the inflaton evolves. Comparing against the helical inflation trajectory for quadratic inflation [9], the deformed path becomes rather steep, finally forcing departure from the inflationary phase, for small $\theta$, while it tends toward extreme flatness for large $\theta$. Taking $c=c_{S} \equiv \frac{2}{\sqrt{3}}$, after a canonical field rescaling $\theta \rightarrow \frac{1}{\sqrt{2}} \theta$, the potential (2.5) exactly reproduces the Starobinsky model. However, there is no implied constraint on $c$, and we do not see a special interpretation of the value $c_{S}$ at this stage. The model described by eqs. (2.1) therefore represents a generalized Starobinsky-like model of inflation. For small $c \rightarrow 0$, it approaches quadratic inflation. More details on the inflationary predictions of this potential are given in figure 3 . These predictions are very well consistent with new experiment data [40-42], as long as the parameter $c$ is not too small.

Interpolation from natural inflation to Starobinsky-like inflation. We have shown that a simple supergravity construction (2.1) can lead to natural inflation if the power of $\Phi$ in $W$ is real, or Starobinsky-like inflation if the power of $\Phi$ is pure imaginary. A natural consideration is the prospect of generalizing the power to arbitrary complex values, as in the following superpotential

$$
W=a \frac{X}{\Phi}\left(\Phi^{\chi}-1\right)
$$

where $\chi=b+i c$. It is easy to show that after field stabilization $X \rightarrow\langle X\rangle=0$, the prior 
superpotential leads to the scalar potential

$$
V(r, \theta)=a^{2} \frac{e^{r^{2}}}{r^{2}}\left(r^{2 b} e^{-2 c \theta}-2 r^{b} \cos (c \log r+b \theta) e^{-c \theta}+1\right) .
$$

Taking a small real exponent, with $c=0$ and $b \ll 1$, this potential stabilizes the field norm $|\Phi| \approx 1$ and the inflaton potential reduces to $V(\theta)=2 a^{2}(1-\cos (b \theta))$, corresponding to natural inflation, as detailed in [10]. Curvature along the radial direction is determined by the coefficient $e^{r^{2}} / r^{2}$, which admits a global minimum at $r=1$ and gives a large mass above Hubble scale. Extra couplings between the field norm $r$ and phase $\theta$ in $V(r, \theta)$ can partially affect the stabilization of $|\Phi|$, although $b \ll 1$ and $e^{-c \theta} \ll 1$ during inflation, such that corrections to observables are of order $o\left(b^{2}\right)$, and can be ignored in a primary evaluation. With stabilized field norm $|\Phi|=1$, but no constraint on $c$, the scalar potential $V(r, \theta)$ becomes

$$
V(\theta)=a^{2}\left(e^{-2 c \theta}-2 \cos (b \theta) e^{-c \theta}+1\right) .
$$

By varying $b$ and $c$ one may cleanly interpolate from natural inflation $(c=0, b \ll 1)$ to Starobinsky-like inflation $(c>0, b=0)$. Parameterizations of the potential corresponding to the quadratic inflation, natural inflation, interpolation inflation $(b c \neq 0)$, and Starobinsky-like inflation scenarios are shown in figure 4. The deformed potentials tend to be flatter in the large field region, and steeper in small field region, indicating weaker tensor fluctuation.

The potential's deformation can be effectively characterized by the complex parameter $\chi \equiv b+i c$. Its real component $\Re(\chi)$ relates to the phase/axion decay constant for natural inflation and its imaginary component $\Im(\chi)$ describes the interpolation between quadratic inflation and Starobinsky inflation, which is, according to the no-scale supergravity realization of Starobinsky-like inflation [13-15, 45], the parameter $n$ in the generalized no-scale type Kähler potential

$$
K=-n \log (\Phi+\bar{\Phi})+f(\Phi)+\bar{f}(\bar{\Phi})
$$

that describes a Kähler manifold with curvature $R=\frac{2}{n}$. The parameter $\Im(\chi)$ is introduced in ref. [44] as a phenomenological generalization of Starobinsky inflation, and it is also the parameter $\alpha$ in language of $\alpha$-attractors [46]. The ratio $\Re(\chi) / \Im(\chi)$ indicates whether the deformed potential for interpolation inflation better approximates either natural inflation or Starobinsky-like inflation. Inflationary predictions of the model (2.6) with different index values $\chi$ are presented in figure 5. As shown in the graph, with fixed e-folding number $N=60$, each point on the $n_{s}-r$ plane [40] within the region favored by new Planck results is coincident with predictions of the generalized inflationary model (2.6) for some specific choice of the index $\chi$.

It is surprising that the simple supergravity model (2.6) can introduce such an abundant variety of results, which effectively characterize the entire $n_{s}-r$ region consistent with new Planck data by modulation of the single complex-valued index $\chi$. However, the origin of this index is a puzzle, and its physical meaning is unclear from the model (2.6). It should also be noted that this model exhibits a pole at $\Phi=0$, and is well-defined only in the large field region $|\Phi| \gg 0$; as such, it should be considered as an effective theory with 


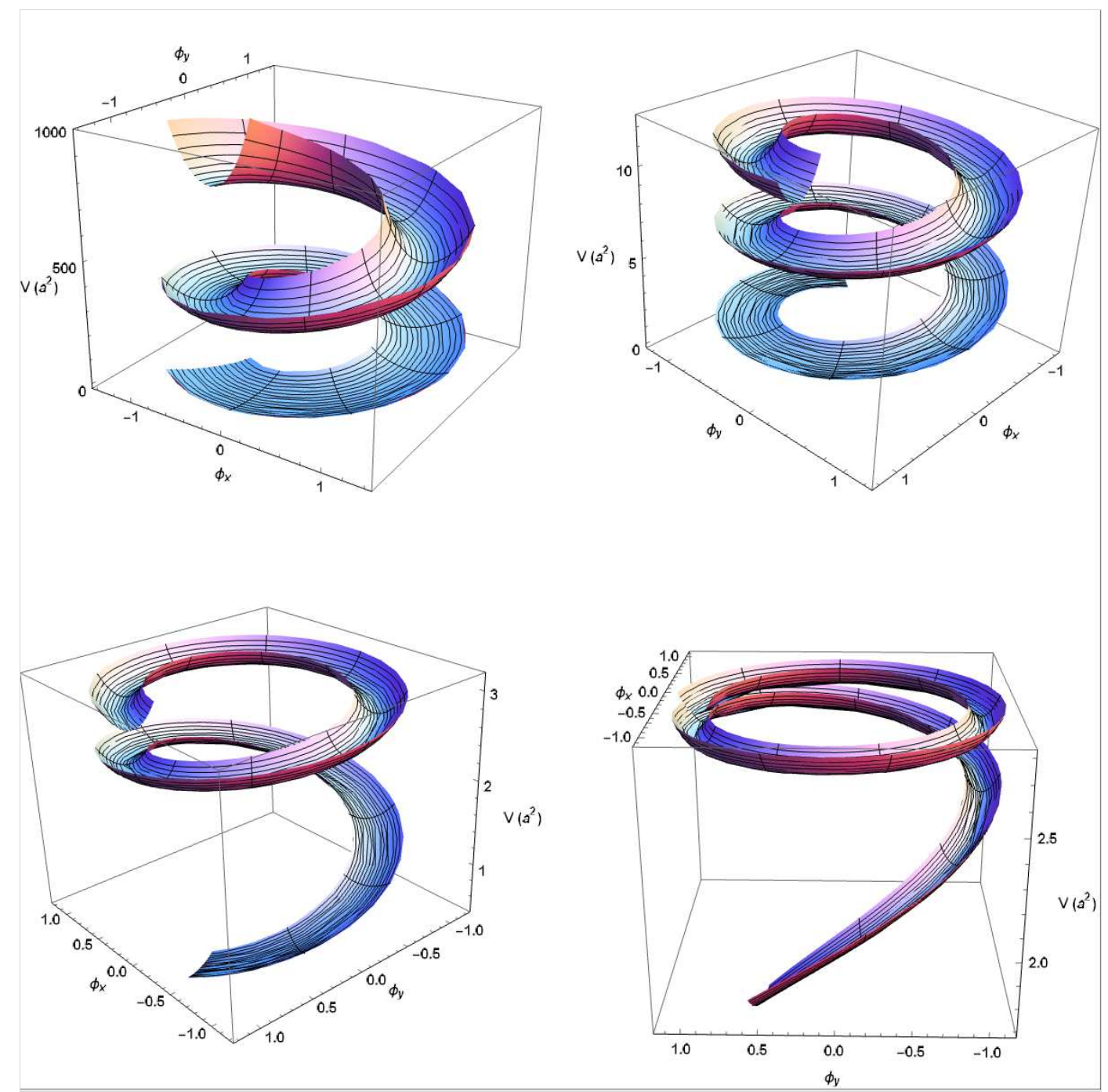

Figure 4. Parameterization of the helicoid potentials associated with quadratic inflation (upperleft), natural inflation (upper-right), interpolation inflation (lower-left), and Starobinsky-like inflation (lower-right) scenarios are depicted. For the later three inflationary models, the parameters $(c, b)$ are selected as $(0,0.15),(0.2,0.15)$, and $(0.6,0)$, respectively. Deformations render the helicoid flatter in the large field region, and steeper in small field region.

heavy fields integrated out. A more fundamental model including heavy fields may help us to unveil the physical meaning of the index $\chi$.

\section{Global U(1) symmetry in the superpotential}

The supergravity construction for helical phase inflation is considered to be an effective theory. The Kähler potential and superpotential are rather simple, while several critical facets of the superpotential, such as the pole at $\Phi=0$ and the phase monodromy, require further elaboration. We follow the method proposed in $[9,10]$, where the phase monodromy 


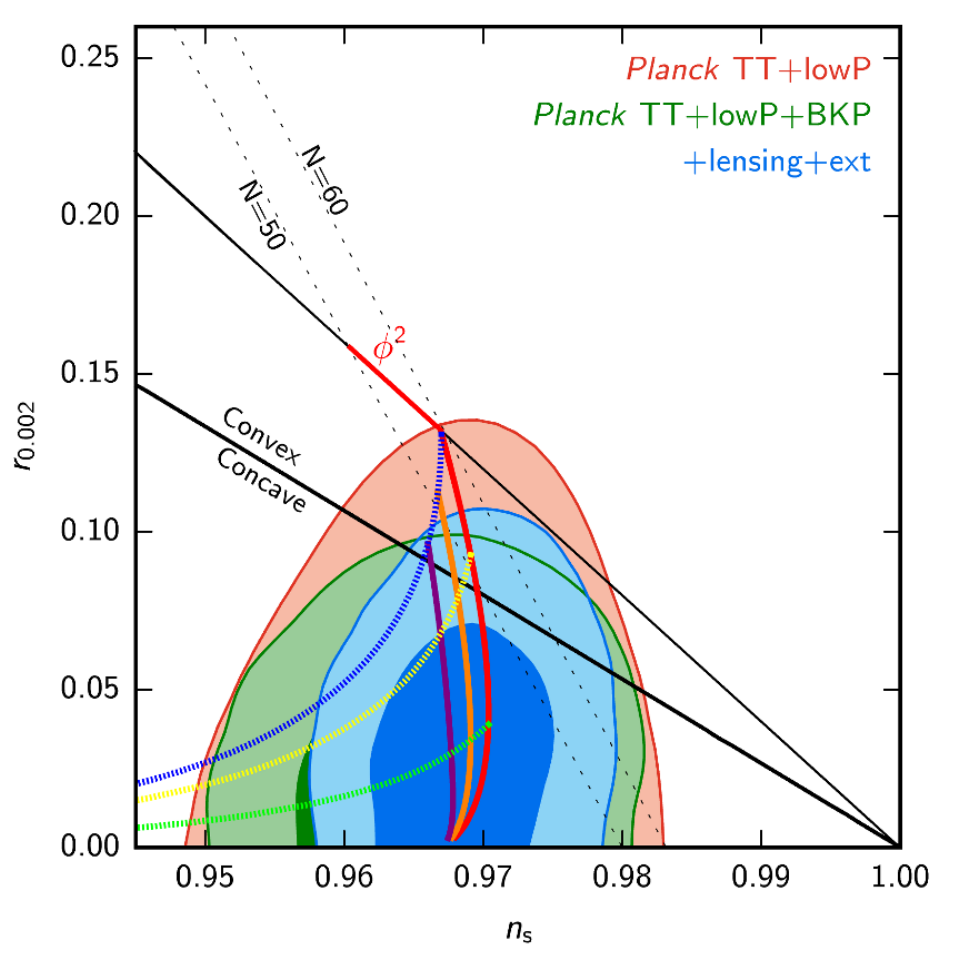

Figure 5. Predictions on $n_{s}-r$ relation of the generalized inflationary model (2.6) with fixed e-folding number $N=60$. Dashed lines (with running $b$ ) represent blue: $c=0$ (natural inflation), yellow: $c=0.05$, and green: $c=0.2$; thick lines (with running $c$ ) represent red: $b=0$ (Starobinskylike inflation), orange: $b=0.1$, and purple: $b=0.14$.

appearing in the superpotential (2.1) and (2.6) is realized by explicitly breaking of a global $\mathrm{U}(1)$ symmetry.

We begin with the following superpotential

$$
W=a X \Psi\left(e^{-\alpha T_{1}}-\rho\right)+Y\left(e^{-\beta T_{2}}-\sigma \Psi\right)+Z(\Phi \Psi-\lambda)+\cdots,
$$

in which the first term $W_{I}=a X \Psi\left(e^{-\alpha T_{1}}-\rho\right)$ is used to generate the inflaton potential, so the coefficient should be rather small $a \ll 1$. Another pair of terms appear at energy scales hierarchically higher than that of inflation, with coupling coefficients significantly larger than $a$, which we presently set to 1 for convenience. Two racetrack-type non-perturbative terms are included. Given $T_{1}=T_{2}$, the superpotential (3.1) reduces to the natural inflation model [10]. The exponentials are multiplied with stabilizer fields $X, Y$ that vanish during inflation and their F-terms provide for the inflaton potential or for field stabilization. The non-perturbative terms are expected to be obtained from D-brane instanton effects. The D-brane instanton effects are widely studied in the construction of matter couplings favored for their phenomenological aspects (more details are provided in [72]). The advantage of the D-brane instanton mechanism is that the magnitudes of its associated terms do not have to be too small and can be applied for field stabilization above the inflation scale. In contrast, the non-perturbative superpotential from gauge theory instantons is usually substantially suppressed and there is not much space to establish a hierarchy between 
inflation and field stabilization. Extra Kähler moduli terms are omitted in (3.1), which are expected to provide linear constraints on $T_{1}$ and $T_{2}$. A detailed study of these terms will be provided later.

The superpotential (3.1) features a global U(1) symmetry. The superfields transform under the $\mathrm{U}(1)$ symmetry

$$
\begin{array}{lll}
X \rightarrow X e^{-i q \theta}, & Y \rightarrow Y e^{-i q \theta}, & Z \rightarrow Z, \\
\Psi \rightarrow \Psi e^{i q \theta}, & \Phi \rightarrow \Phi e^{-i q \theta}, & T_{2} \rightarrow T_{2}-i \frac{q}{\beta} \theta,
\end{array}
$$

Given $T_{1}=T_{2}$, the superpotential (3.1) reduces to the natural inflation model [10]. The $\mathrm{U}(1)$ symmetry is explicitly broken by the inflation term $W_{I}$, while we can assume in this model that $T_{1}$ is neutral under $\mathrm{U}(1)$. A global U(1) symmetry of this kind appears in the Kähler potential more naturally. For the matter fields, their Kähler potentials are of the minimal type, and invariant under U(1) transformation. The Kähler modulus $T_{2}$ shifts under $\mathrm{U}(1)$, and its Kähler potential is of no-scale type, which is thus independent of the imaginary component. Consequently, the global U(1) symmetry is inherited by the F-term scalar potential, forming an exactly flat direction. To lift the flat direction one has to break the global U(1) symmetry, which can be achieved by applying a linear constraint between two Kähler moduli

$$
T_{1}-\kappa T_{2}+\delta=0
$$

Here, we require the constraint to satisfying the conditions $\Re\left(T_{1}\right)>0$ and $\Re\left(T_{2}\right)>0$, since the real components of $T_{i}$ give the volumes of internal cycles. We will show that such linear constraint on Kähler moduli are common in non-geometric flux compactification, and that the coefficient $\kappa$ has a clear geometric origin.

The flat direction is lifted after U(1) symmetry breaking, leaving a unique Minkowski vacuum. To isolate the vacuum, we need to solve the equations:

$$
W=D_{z} W=W_{z}+K_{z} W=W_{z}=0,
$$

where $z \in\left\{\Phi, \Psi, X, Y, Z, T_{1}, T_{2}\right\}$. It is easy to show that the superpotential (3.1) admits a supersymmetric Minkowski vacuum at

$$
\begin{array}{ll}
X=Y=Z=0, & T_{1}=-\frac{\ln \rho}{\alpha}, \quad T_{2}=\left\langle T_{2}\right\rangle=-\frac{\ln \rho}{\alpha \kappa}+\frac{\delta}{\kappa}, \\
\Psi=\frac{1}{\sigma} e^{-\beta\left\langle T_{2}\right\rangle}, & \Phi=\sigma \lambda e^{\beta\left\langle T_{2}\right\rangle} .
\end{array}
$$

The parameters are manually adjusted such that $\langle|\Phi|\rangle \gg\langle|\Psi|\rangle$. The fields $Y, Z, \Psi, T_{2}$ obtain masses significantly above the inflationary energy scale according to the superpotential (3.1), whereas $T_{1}$ is limited by the prior constraint. During inflation these degrees of freedoms are thus frozen and should be integrated out. To integrate out heavy fields, we should solve F-term equations. Non-trivial results are obtained from the F-term equations associated with the stabilizer fields $Y$ and $Z$ :

$$
\begin{aligned}
& F_{Y}=D_{Y} W=e^{-\beta T_{2}}-\sigma \Psi+\bar{Y} W \approx e^{-\beta T_{2}}-\sigma \Psi=0, \\
& F_{Z}=D_{Z} W=\Phi \Psi-\lambda+\bar{Z} W \approx \Phi \Psi-\lambda=0,
\end{aligned}
$$


where we have ignored terms proportional to $\bar{Y}$ or $\bar{Z}$, given that these fields are strongly stabilized at $\langle Y\rangle=\langle Z\rangle=0$ during inflation. The heavy fields are solved for in term of $\Phi$

$$
\begin{aligned}
\Psi & =\frac{\lambda}{\Phi}, \\
e^{-\beta T_{2}} & =\frac{\sigma \lambda}{\Phi}, \\
e^{-\alpha T_{1}} & =e^{\alpha \delta}(\sigma \lambda)^{\frac{\alpha \kappa}{\beta}} \Phi^{-\frac{\alpha \kappa}{\beta}},
\end{aligned}
$$

and the effective low energy theory becomes

$$
W=a \lambda \frac{X}{\Phi}\left(e^{\alpha \delta}(\sigma \lambda)^{\frac{\alpha \kappa}{\beta}} \Phi^{-\frac{\alpha \kappa}{\beta}}-\rho\right),
$$

which is the superpotential (2.6) for helical phase inflation, with a suitable parameter redefinition. The index $\chi$ is given by $\chi=-\frac{\alpha}{\beta} \kappa$, where $\alpha$ and $\beta$ are positive parameters and only affect the magnitude of $\chi$.

\section{Moduli constraint from flux compactification}

Constraints on Kähler moduli in type IIB compactification, or on the T-dual complex structure moduli in type IIA compactification, are obtained from moduli stabilization, which requires Kähler moduli couplings in the superpotential. However, no perturbative term on Kähler moduli can be generated from type IIB compactification with RR or NSNS fluxes. By contrast, they do appear in type IIA compactifications with geometric fluxes and also in type IIB compactifications with non-geometric fluxes. Both geometric and non-geometric fluxes arise from T-duality of NSNS 3-form fluxes.

\subsection{Type IIA compactification with geometric fluxes}

T-duality connects IIB and IIA orientifold compactifications. The linear constraint on Kähler moduli in IIB compactification is T-dualized to a linear constraint on complex structure moduli in IIA. For type IIB orientifold compactification with NSNS flux $H_{i j k}$, taking T-duality along a direction $x_{i}$ of the internal space $M$, the flux $H_{i j k}$ is mapped into a new type of flux. This is the geometric flux $\omega_{j k}^{i}$, which is equivalent to IIA compactification on a twisted torus. The twisted torus is described by the geometric flux $\omega_{j k}^{i}$ as

$$
d \eta^{i}=-\frac{1}{2} \omega_{j k}^{i} \eta^{j} \wedge \eta^{k}
$$

where $\omega_{j k}^{i}=-\omega_{k j}^{i}$ and $\eta^{j}$ are tangent 1-forms linearly depending on the internal coordinates $x^{j}$. The dual tangent vectors $Z_{i}$ of the 1 -form $\eta^{i}$ form a Lie algebra with geometric fluxes $\omega_{j k}^{i}$ as the structure constants

$$
\left[Z_{i}, Z_{j}\right]=\omega_{i j}^{k} Z_{k}
$$

Consequently, the fluxes should satisfy the Jacobi identity

$$
\omega_{[j k}^{i} \omega_{m] n}^{l}=0 .
$$


For IIA toroidal compactification with O6-planes and geometric fluxes $\omega$, the orientifold action is $\Omega(-1)^{F_{L}} R_{A}$ and the involution $R_{A}$ acts on the local internal complex coordinates $z_{i}$ as $R_{A}\left(z_{i}\right)=\bar{z}_{i}$. In the low-energy effective theory, the NSNS and geometric fluxes generate following terms in superpotential

$$
W_{N S \& \omega}=\int_{T_{6}} \Omega_{c} \wedge\left(H_{3}+d J_{c}\right)
$$

where $\Omega_{c}$ and $J_{c}$ are a holomorphic 3-form and Kähler 2-form, respectively. Also, the RR flux superpotential is of the typical form

$$
W_{R R}=\int_{T_{6}} e^{J_{c}} \wedge F_{R R}
$$

where the $F_{R R}$ are RR fluxes. Combining $W_{N S \& \omega}$ and $W_{R R}$, the full superpotential reads

$$
W\left(T_{i}, S, U_{i}\right)=P_{-1}\left(T_{i}\right)+S P_{0}\left(T_{i}\right)+\sum_{k=1}^{3} U_{k} P_{k}\left(T_{i}\right),
$$

where $P_{-1}$ is cubic on $T_{i}$, while $P_{0}, P_{i}$ are linear on $T_{i}$. Geometric flux quanta as coefficients in $W$ are subjected to the Jacobi identity constraint (4.3). The tree level Kähler potential is of no-scale type

$$
K=-\log (S+\bar{S})-\sum_{i=1}^{3} \log \left(U_{i}+\bar{U}_{i}\right)-\sum_{i=1}^{3} \log \left(T_{i}+\bar{T}_{i}\right) .
$$

We are interested in obtaining a supersymmetric Minkowski vacuum from $W$. Supersymmetric Minkowski vacuum equations (4.3) for $S$ and $U_{i}$, together with the condition $W=0$, require

$$
P_{-1}\left(T_{i}\right)=P_{0}\left(T_{i}\right)=P_{i}\left(T_{i}\right)=0 .
$$

In principle, the three Kähler moduli can be solved for from the prior equations. However, there are more equations than variables, so mutual consistency is not guaranteed. On the other hand, we do have substantial freedom to turn on fluxes, and the two extra equations are equivalent to non-linear constraints on these flux coefficients. Since the fluxes are quantized, only integer solutions of these constraints are physical. Additionally, there are three equations associated with the supersymmetric Minkowski vacuum for $T_{i}$

$$
\partial_{T_{i}} P_{-1}+S \partial_{T_{i}} P_{0}+\sum_{k=1}^{3} U_{k} \partial_{T_{i}} P_{k}=0
$$

with 4 variables $S$ and $U_{k}$. Through variable elimination, one obtains a constraint relating two moduli, of the type needed in eq. (3.3).

However, both $P_{0}$ and $P_{i}$ are linear on $T_{i}$, and their coefficients are from flux quanta, and thus real. The expressions in (4.9) give a moduli constraint corresponding to a real parameter $\kappa$ in eq. (3.3), yielding a real value for the index $\chi$, which produces natural inflation. This is equivalent to the simple isotropy condition stating that the three sub-tori of $T^{6}=T^{2} \times T^{2} \times T^{2}$ admit exchange symmetry with $T_{i}=T$, and $U_{i}=U$. To obtain more general moduli constraints with complex coefficients and a generically complex $\kappa$, we need $P_{0}$ or $P_{i}$ to exhibit high-order couplings. This is realized by introducing non-geometric Q-fluxes, which are T-duals of the geometric flux $\omega$. 


\subsection{Type IIB compactification with Q-fluxes}

By taking successive T-dualities along different compact coordinates, more fluxes arise without geometric interpretation, which are dubbed non-geometric fluxes. These fluxes are related to each other under T-duality

$$
H_{i j k} \stackrel{T_{i}}{\longleftrightarrow} \omega_{j k}^{i} \stackrel{T_{j}}{\longleftrightarrow} Q_{k}^{i j} \stackrel{T_{k}}{\longleftrightarrow} R^{i j k},
$$

where $T_{i}$ refers to the T-duality along compact direction $x^{i}$. In this section, we study type IIB orientifold compactification on Calabi-Yau manifolds $M$ with an orientifold projection $\Omega_{P}(-1)^{F_{L}} R_{B}$, in which $\Omega_{P}$ is the world-sheet parity operator, $F_{L}$ is the left-moving fermion number and $R_{B}$ is the orientifold involution. The superpotential from non-geometric fluxes contains Kähler moduli couplings at the perturbative level and admits rich vacuum configurations.

For type IIB orientifold compactification with O3-planes, the orientifold involution $R_{B}$ acts on compact coordinates $x_{i}$ as $R_{B}: x_{i} \rightarrow-x_{i}$, and its actions on the Kähler form $J$ and the holomorphic 3 -form $\Omega_{3}$ are therefore

$$
R_{B}(J)=J, \quad R_{B}\left(\Omega_{3}\right)=-\Omega_{3} .
$$

The involution $R_{B}$ projects out even parts of the cohomology $H^{3}(M)$ and odd parts of the cohomologies $H^{1,1}(M)$ and $H^{2,2}(M)$. Remaining cohomologies are denoted as

$$
\begin{aligned}
\omega_{i} & \in H_{+}^{1,1}(M) & & i=1, \cdots, h_{+}^{1,1} \\
\tilde{\omega}^{i} & \in H_{+}^{2,2}(M) & & i=1, \cdots, h_{+}^{1,1} \\
\left\{\alpha_{m}, \beta^{m}\right\} & \in H_{-}^{3}(M) & & m=0, \cdots, h_{-}^{2,1} .
\end{aligned}
$$

The holomorphic 3-form $\Omega_{3}$ can be expanded in terms of the symplectic basis of $H_{-}^{3}(M)$

$$
\Omega_{3}=X^{m} \alpha_{m}-F_{m} \beta^{m},
$$

where $X^{m}=\int_{A^{m}} \Omega_{3}$ and $F_{m}=\int_{B_{m}} \Omega_{3}$ are periods of the compactification manifold $M$ with a symplectic three cycle basis $\left\{A^{m}, B_{m}\right\}$. Taking $X^{m}$ as coordinates on the complex structure moduli space, periods $F_{m}$ can be represented as partial derivatives on the prepotential $F=f_{i j k} X^{i} X^{j} X^{k} / X^{0}$, specifically $F_{m}=\partial_{X_{m}} F$. The low-energy effective theory is described by $N=1$ supergravity with a tree level Kähler potential

$$
K=-\log \left(-i \int_{M} \Omega_{3} \wedge \bar{\Omega}_{3}\right)-\log (S+\bar{S})-2 \log \left(e^{-\frac{3}{2} \phi} \int_{M} J \wedge J \wedge J\right) .
$$

The superpotential is given by the generalized Gukov-Vafa-Witten superpotential [69]

$$
W=\int_{M}\left(F_{3}-i S H+i T_{i}\left(Q \bullet \tilde{\omega}^{i}\right)+\cdots\right) \wedge \Omega_{3},
$$

where the $Q$ action on the $p$-form $F_{M_{1} \cdots M_{p}}$ gives the $p-1$-form

$$
(Q \bullet F)_{N M_{1} \cdots M_{p-2}}=\frac{1}{2} Q_{[N}^{J K} F_{\left.M_{1} \cdots M_{p-2}\right] J K},
$$


leading to Q-flux terms in the superpotential that depend linearly on the Kähler moduli $T_{i}$. The 4 -forms $\tilde{\omega}^{i}$ are a basis of even $(2,2)$-cohomology. The first two terms in (4.15) are just the well-known Gukov-Vafa-Witten superpotential [73] for NSNS and RR fluxes, namely $F_{3}=d C_{2}$ and $H=d B_{2}$. In the superpotential $W$ we have ignored the geometric and R-type fluxes that could also play an interesting role in moduli stabilization. The R-type fluxes do not appear in the superpotential due to the symmetry under orientifold projection but they can involve in the D-terms potential [74-76]. More details on these fluxes are provided in ref. [69].

Expanding the $p$-form fluxes on a cohomology basis with arbitrary flux quanta, the superpotential $W$ can be expressed in terms of flux quanta and moduli

$$
W=-\left(e_{m} X^{m}-\tilde{e}^{m} F_{m}\right)+i S\left(a_{m} X^{m}-\tilde{a}^{m} F_{m}\right)+i T_{i}\left(b_{m i} X^{m}-\tilde{b}^{m i} F_{m}\right)+\cdots .
$$

The superpotential $W$ implicitly depends on the complex structure moduli $U^{m}$ through the $H_{-}^{2,1}(M)$ periods $X^{m}$ :

$$
U^{m}=-i \frac{X^{m}}{X^{0}}
$$

After turning on non-geometric fluxes, the Lie algebra (4.2) is extended with new generators. The NSNS, geometric and non-geometric fluxes become structure constants of the extended Lie algebra, and the Jacobi identities of the Lie algebra introduce new constraints on fluxes $[60,61,69]$. Additionally, these fluxes also contribute to the RR 4 -form and 8-form tadpoles, which should satisfy the tadpole cancellation conditions in conjunction with the O3/D3 and O7/D7 contributions.

The superpotential in eq. (4.17) contains perturbative couplings of the Kähler moduli. In contrast with the case of type IIA orientifold compactification with geometric fluxes, the complex structure terms $b_{m i} X^{m}-\tilde{b}^{m i} F_{m}$ coupled with Kähler moduli are not just linear, but are up to third order, leading to more interesting moduli stabilization and vacuum configurations. The supersymmetric Minkowski vacuum and moduli stabilization from the Type IIB compactifications on orientifolds have been studied in refs. [61-67]. Most of these works targeted the compactification on isotropic $T^{6}$, i.e., with an exchange symmetry among three sub-tori so that $T_{1}=T_{2}=T_{3}$ and $U_{1}=U_{2}=U_{3}$. In our study, the multiKähler and complex structure moduli are needed. We want to realize constraints between Kähler moduli instead of fixing all the Kähler moduli completely. Without the isotropy constraint, there is additional freedom to adjust the flux quanta, producing richer vacuum configurations. However, with more Kähler and complex structure moduli, the Jacobi identities for fluxes become extremely clumsy. The Jacobi identities, together with the tadpole cancellation conditions and equations for the supersymmetric Minkowski vacuum, could be solved by numerically scanning the parameter space. Mathematical techniques from algebraic geometry have been applied to solve the flux constraints and supergravity equations using the programs Mathematica and Singular [77]. In this work, we propose a toy model with a superpotential like that in eq. (4.17) to show how the constraint on Kähler moduli appears through moduli stabilization in the supersymmetric Minkowski vacuum. However, in this toy model we do not expect to solve the Jacobi identities with given fluxes. We will discuss the effects of these Jacobi identities on the Minkowski vacua later. 
We consider the Type IIB orientifold compactification on a Calabi-Yau manifold with $h_{+}^{1,1}=2, h_{-}^{1,1}=0, h_{-}^{1,2}=3$. In this model, the Kähler potential is

$$
K=-\sum_{i=1}^{3} \log \left(U_{i}+\bar{U}_{i}\right)-\log (S+\bar{S})-\log \left(T_{1}+\bar{T}_{1}\right)-2 \log \left(T_{2}+\bar{T}_{2}\right),
$$

where we have implicitly assumed that in the prepotential $F$ the only non-vanishing component of symmetric coefficients $f_{i j k}$ is $f_{123}=1$. The fluxes are adjusted to generate following superpotential

$$
\begin{aligned}
W & =W_{\mathrm{NSR}}+W_{Q}, \\
W_{\mathrm{NSR}} & =e_{0}+q_{1} U_{2} U_{3}+a_{1} S U_{1}+a_{2}\left(U_{1}+S\right)\left(U_{2}+U_{3}\right)+\tilde{h} S U_{1} U_{2} U_{3}, \\
W_{Q} & =\left(U_{2}-U_{3}\right)\left(b T_{1}-i \tilde{b} T_{2} U_{1}\right) .
\end{aligned}
$$

The superpotential $W_{\text {NSR }}$ admits exchange symmetries in terms of $U_{2} \leftrightarrow U_{3}$ and $S \leftrightarrow U_{1}$. Only even order couplings among $S$ and $U_{i}$ are considered, so that the flux quanta appear in $W_{\text {NSR }}$ as real coefficients. These limitations on the superpotential are not necessary for a supersymmetric Minkowski vacuum, but make the calculations sufficient simple for an example. Another term $W_{Q}$ is quadratic in $U_{i}$ and is expected to be obtained from $Q$-fluxes, which can generate couplings of the complex structure moduli up to third order, as shown in eq. (4.17). Again we remind the readers that above potentials should be considered as an example to show the linear constraint of Käher moduli obtained as relic of moduli stabilization, instead of generating a "physical" Minkowski vacuum since not all Jacobi identities are fully satisfied with given fluxes and prepotential coefficient.

It is straightforward to show that the superpotential $W_{\mathrm{NSR}}$ yields a supersymmetric Minkowski vacuum [78], i.e. that

$$
W_{\mathrm{NSR}}=\partial_{U_{i}} W_{\mathrm{NSR}}=\partial_{S} W_{\mathrm{NSR}}=0
$$

at

$$
\begin{aligned}
S & =U_{1}=\sqrt{\frac{q_{1}}{a_{1} \tilde{h}}}\left(-a_{1} \pm 2 a_{2} \sqrt{\frac{a_{1}}{q_{1}}}\right)^{1 / 2}, \\
U_{2} & =U_{3}= \pm \sqrt{\frac{a_{1}}{q_{1}}} S, \quad e_{0}=\frac{q_{1}}{a_{1} \tilde{h}}\left(-a_{1} \pm 2 a_{2} \sqrt{\frac{a_{1}}{q_{1}}}\right)^{2} .
\end{aligned}
$$

Taking the flux quanta $\left(e_{0}, a_{1}, a_{2}, q_{1}, \tilde{h}\right)=(2,2,-2,2,2)$, and ignoring unphysical solutions, one can realize a supersymmetric vacuum at $S=U_{i}=1$. Examples with complex vacuum expectation values of $U_{i}$ are also provided in [78].

If the $Q$-fluxes introduce perturbative couplings $W_{Q}$ of Kähler moduli, then the equations for a supersymmetric Minkowski vacuum become

$$
\begin{aligned}
\partial_{S} W_{\mathrm{NSR}}=W_{\mathrm{NSR}}+\left(U_{2}-U_{3}\right)\left(b T_{1}-i \tilde{b} T_{2} U_{1}\right) & =0, \\
\partial_{T_{1}} W_{Q}=\partial_{T_{2}} W_{Q}=U_{2}-U_{3} & =0, \\
\partial_{U_{1}} W_{\mathrm{NSR}}-i \tilde{b} T_{2}\left(U_{2}-U_{3}\right) & =0, \\
\partial_{U_{2}} W_{\mathrm{NSR}}+\left(b T_{1}-i \tilde{b} T_{2} U_{1}\right) & =0, \\
\partial_{U_{3}} W_{\mathrm{NSR}}-\left(b T_{1}-i \tilde{b} T_{2} U_{1}\right) & =0 .
\end{aligned}
$$


It is easy to see that above equations are equivalent to the equations of $W_{\mathrm{NSR}}$ (4.21) plus an extra constraint on the Kähler moduli

$$
b T_{1}-i \tilde{b} T_{2}\left\langle U_{1}\right\rangle=0 .
$$

According to the moduli stabilization from $W_{\mathrm{NSR}}$, namely $\left\langle U_{1}\right\rangle=1$, this constraint enforces an imaginary ratio $\kappa=i \tilde{b}\left\langle U_{1}\right\rangle / b$ and yields an imaginary index $\chi$, which produces Starobinsky-like helical phase inflation. For the models with complex $\left\langle U_{1}\right\rangle$, we may realize a complex index $\chi$ as well, which produces interpolation inflation. However, the pure imaginary $\left\langle U_{1}\right\rangle$ for natural inflation corresponds to a boundary of the complex structure moduli space. This solution is not physical, as it indicates a degenerate internal space.

The overall superpotential contains term associated with both the string moduli (4.20) and the helical phase inflation supergravity construction (3.1), and the latter also depend on the Kähler moduli through non-perturbative effects. Therefore, non-perturbative terms appear in equations $\partial_{T_{i}} W=0$ as well, and may affect the vacuum equations $\partial_{T_{i}} W=$ $U_{2}-U_{3}=0$ that are necessary to facilitate the exchange symmetry $U_{2} \leftrightarrow U_{3}$ in $W_{\mathrm{NSR}}$. Fortunately, this is avoided due to vanishing of the stabilizer fields $X$ and $Y$ in (3.1). The string moduli stabilization and generation of dynamics for helical phase inflation are therefore reducible, even though the Kähler moduli appear in both sets of equations. It is easy to generalize the $Q$-flux superpotential $W_{Q}$ to get different Kähler moduli constraints. Discrete symmetries are employed here, and they play an important role in simplifying calculations. Since there are more equations than variables, these discrete symmetries help to maintain mutual consistency of the equations. For a more realistic model, i.e. one combining the vacuum equations with Jacobi identities and RR 4-form $C_{4}, 8$-form $C_{8}$ tadpole constraints, the calculations become quite cumbersome, and it is necessary to scan the parameter space numerically in order to identify realistic vacua that satisfy the formalism and provide a linear constraint on Kähler moduli. In fact, such a constraint on the Kähler moduli is a natural outcome for the supersymmetric Minkowski vacua.

It is interesting to compare our proposal with the work in [58], which also studied the Starobinsky-like inflation based on non-geometric flux compactification. In [58] the inflaton is from string moduli which strongly interact with other heavy fields, so it needs to adjust the parameters carefully so that the inflation direction is sufficient flat while all the extra fields are at or above Hubble scale. Because for large field inflation a super-Planckian field excursion is required and the inflation dynamics is rather sensitive to the super-Planckian physics, one also needs to check whether the large field inflation can be reasonably studied in the low energy effective theory. In particular the following hierarchy should be unbroken under super-Planckian string moduli/axion field excursion [58]

$$
M_{P}>M_{s}>M_{\mathrm{KK}}>M_{\text {moduli }}>H_{\text {inflation }}>M_{\text {inflaton }}
$$

Interestingly, in helical phase inflation, the flatness condition of inflation potential is protected by a mildly broken U(1) symmetry, and the UV-completion problem of large field inflation is avoided. Thus, we do not need to struggle with the $\eta$ problem or the effectiveness of the low energy theory obtained from string compactification. Moreover, besides 
the normal conditions on string compactification, the above hierarchy condition (4.25) is replaced by a single hierarchy in eq. (3.1): $a \ll 1$. In other words, the first term in eq. (3.1) is significantly smaller than the other terms so that we can safely integrate them out. In contrast, in our proposal the non-geometric fluxes lead to much more complex constraints than those in [58]. We expect the constraints from the flux Jacobi identities and Minkowski vacuum conditions can be solved numerically.

Supersymmetric Minkowski vacua with a constraint on the Kähler moduli are expected to appear from type IIB orientifold compactification on $T^{6} / \Omega_{P}(-1)^{F_{L}} R_{B}$. Such compactifications have been studied in refs. [61-69] as a mechanism for obtaining vacua with full moduli stabilization. The authors of these works have mainly focused on the simplified case where an exchange symmetry exists among three sub-tori. The associated results are very limited unless a non-geometric $P$-flux arising from S-duality of type IIB string theory is also introduced. In our case, with loose moduli stabilization criteria and no exchange symmetry, there is more freedom to arrange the flux quanta in order to obtain supersymmetric Minkowski vacua. The superpotential for NSNS, RR and $Q$-type fluxes is

$$
W\left(U_{i}, S, T_{i}\right)=P_{-1}\left(U_{i}\right)+S P_{0}\left(U_{i}\right)+\sum_{k=1}^{3} T_{k} P_{k}\left(U_{i}\right),
$$

where the terms $P_{0}$ and $P_{i}$ for NSNS 3-form and Q-fluxes are cubic in $U_{i}$ :

$$
\begin{aligned}
P_{-1} & =f_{0}+i \sum_{i=1}^{3} f_{i} U_{i}-\sum_{i=1}^{3} \frac{\tilde{f}_{i}}{U_{i}} U_{1} U_{2} U_{3}+i \tilde{f}_{0} U_{1} U_{2} U_{3}, \\
P_{0} & =i g_{0}-\sum_{i=1}^{3} g_{i} U_{i}+i \sum_{i=1}^{3} \frac{\tilde{g}_{i}}{U_{i}} U_{1} U_{2} U_{3}-\tilde{g}_{0} U_{1} U_{2} U_{3}, \\
P_{k} & =-i h_{k}-\sum_{i=1}^{3} h_{i k} U_{i}+i \sum_{i=1}^{3} \frac{\tilde{h}_{i k}}{U_{i}} U_{1} U_{2} U_{3}+\tilde{h}_{k} U_{1} U_{2} U_{3} .
\end{aligned}
$$

The supersymmetric Minkowski vacuum equations $W=\partial_{S} W=\partial_{T_{i}} W=0$ require

$$
P_{-1}=P_{0}=P_{i}=0 \text {. }
$$

As is the case for type IIA orientifold compactification, these equations give vacuum expectation values for $U_{i}$ and also certain non-linear constraints on flux quanta. There are still three vacuum equations from $U_{i}\left(\partial_{U_{i}} W=0\right)$ with four undetermined moduli $S$ and $T_{i}$. After variable elimination, we get a linear constraint on two of the Kähler moduli. Since $P$ s are cubic in $U_{i}$ with both real and imaginary coefficients, generically the ratio of Kähler moduli in the constraint is complex. The non-geometric flux compactification provides an interesting correlation with helical phase inflation. Specifically, for type IIB orientifold compactification on $T^{6} / \Omega_{P}(-1)^{F_{L}} R_{B}$, the supersymmetric Minkowski vacua are consistent with helical phase inflation equipped with a certain index $\chi$.

Given the relationship between Type IIB orientifold compactification with nongeometric fluxes and helical phase inflation, it is interesting to study the distribution of 
supersymmetric Minkowski vacua in the moduli space. Proceeding, we assume that each type of flux quanta forms a direction of the moduli space. Flux quanta are integral, and the vacua are thus located on a lattice of the moduli space. Possible values of the index $\chi$ are uniquely determined in each vacuum, and are therefore discrete points distributed on the complex plane. The magnitude of the index $\chi$ also depends upon non-perturbative effects that may be computed from details of the D-brane configurations. We have shown that the index $\chi$ characterizes the $n_{s}-r$ plane with fixed e-folding number $N$. In principle, predictions for the $n_{s}-r$ relation from helical phase inflation should then likewise correspond to isolated points in the $n_{s}-r$ plane, significantly reducing associated uncertainties. Unfortunately, the preferred e-folding number window $N \in[50,60]$ introduces an uncertainty $\Delta N \approx 10$, which disperses predictions for the $n_{s}-r$ relation across in a small region, even when the index $\chi$ is fixed.

The realization of a distribution of supersymmetric Minkowski vacua from nongeometric flux compactifications can be considered as a generalization of the systematic study of the vacua emerging from type IIB compactification with NSNS and RR fluxes in ref. [79]. The objectives of that work are determination supersymmetric Minkowski vacua fraction favored by low scale supersymmetry and isolation of their corresponding discrete symmetries. The authors focus mainly on $T^{6}$ compactifications with exchange symmetry among sub-tori. In [79], techniques from number theory are employed in order to enumerate the vacua according to the integral nature of flux quanta. Even though the equations for supersymmetric Minkowski vacua ( $W=\partial_{U_{i}} W=0$ in this case) provide strict limitations, there are still abundant distinct solutions corresponding to variation of the flux quanta. In their work, the Kähler moduli do not appear in superpotential. In our case, non-geometric fluxes are introduced, which are constrained by Jacobi identities. Equations for the determination of vacua are similar in both cases, although the variety of solutions is expected to be richer in our model, given the absence of exchange symmetry among sub-tori. On the other hand, the calculations become dramatically more involved in this case. In this work, we have shown that beyond the motivations discussed in [79], the distribution of supersymmetric Minkowski vacua is also deeply related to the extremely important inflationary epoch of our universe. Therefore, a systematically study of the supersymmetric Minkowski vacuum distribution is also very important.

\section{Conclusions}

We have shown that Starobinsky-like inflation can be simply realized as a new type of helical phase inflation, with predictions that are in perfect agreement with new observations from Planck. The advantages of helical phase inflation are inherited in this new model. The so-called $\eta$-problem is directly solved by the global U(1) symmetry built into the Kähler potential of $N=1$ minimal supergravity. Helical phase inflation is driven by the phase component of a complex field, and super-Planckian field excursions are realized within a helical phase rotation, whereas physical fields avoid evolution into the super-Planckian regime where quantum gravitational effects are likely to upset the slow-roll criteria. For Starobinsky-like inflation, even though the tensor-to-scalar ratio $r$ is generically rather 
small $(r \leqslant 0.01)$, the inflaton excursion remains significantly larger the Planck mass during inflation, implying that the treatment of quantum gravitational effects in a suitable UV-completion remains an open problem. This situation is avoided in helical phase inflation with a deformed helicoid potential, where excursions in the radial field direction are strongly stabilized, and evolution proceeds along the trajectory of an extended flat helical minimum. Moreover, as a PNGB, the inflaton is not expected to admit higher polynomial corrections at all.

We have identified an interpolation wherein the helicoid potential can be continuously deformed from natural inflation parameterized by phase/axion decay constant to Starobinsky-like inflation parameterized by $\alpha$-parameter. In helical phase inflation, the interpolation is uniquely characterized by a complex-valued index $\chi$. The helical phase inflation model equipped with index $\chi$ exhibits unexpectedly rich inflationary predictions. The full region of the $n_{s}-r$ plane favored by recent Planck observations can be characterized by variation of the index $\chi$ with a fixed e-folding number $N$.

In the supergravity helical phase inflation construction, the flat phase potential is provided by phase monodromy in the superpotential. The phase monodromy and associated pole at the field-space origin indicate that the superpotential is an effective theory obtained after integrating out heavy fields. We have studied the realization of such phase monodromy based on race-track non-perturbative couplings from D-brane instanton effects. The phase monodromy relates to a global U(1) symmetry in the superpotential that is broken at the inflation scale. The Kähler moduli appearing in non-perturbative terms play an important role in generation of the complex-valued index $\chi$ for helical phase inflation. In turn, they emerge in string moduli stabilization from non-geometric flux compactification.

The index $\chi$ has an interesting geometrical origin. In order to integrate out heavy fields, a linear constraint is required that relates two Kähler moduli appearing in the race-track non-perturbative couplings. Constraint on the Kähler moduli may be realized by the assumption of isotropy $\left(T_{i}=T\right)$, or from type IIA orientifold compactification with NSNS, RR and geometric fluxes. Such constraints have real coefficients and lead to natural inflation. More general constraints with complex coefficients can be obtained by turning on non-geometric fluxes, which generate the requisite higher-order perturbative couplings among Kähler moduli and complex structure moduli. The index $\chi$ is fixed by the vacuum expectation values of complex structure moduli and flux quanta. We suggest a systematic study on the distribution of supersymmetric Minkowski vacua from nongeometric flux compactification in order to obtain viable discrete values of the index $\chi$. This could potentially provide more precise predictions for inflationary observables. Conversely, it could be used to extrapolate geometric structure of the internal space from experimental observations of the inflationary epoch.

\section{Acknowledgments}

We are grateful to J. Walker for valuable comments on the manuscript. The work of DVN is supported in part by the DOE grants DE-FG03-95-ER-40917 and DE-FG02-13ER42020. The work of TL is supported in part by by the Natural Science Foundation of China under grant numbers 11135003, 11275246, and 11475238 . 
Open Access. This article is distributed under the terms of the Creative Commons Attribution License (CC-BY 4.0), which permits any use, distribution and reproduction in any medium, provided the original author(s) and source are credited.

\section{References}

[1] A.A. Starobinsky, A new type of isotropic cosmological models without singularity, Phys. Lett. B 91 (1980) 99 [INSPIRE].

[2] D. Kazanas, Dynamics of the universe and spontaneous symmetry breaking, Astrophys. J. 241 (1980) L59 [INSPIRE].

[3] K. Sato, First order phase transition of a vacuum and expansion of the universe, Mon. Not. Roy. Astron. Soc. 195 (1981) 467.

[4] K. Sato, Cosmological baryon number domain structure and the first order phase transition of a vacuum, Phys. Lett. 99B (1981) 66.

[5] A.H. Guth, The inflationary universe: a possible solution to the horizon and flatness problems, Phys. Rev. D 23 (1981) 347 [INSPIRE].

[6] A.D. Linde, A new inflationary universe scenario: a possible solution of the horizon, flatness, homogeneity, isotropy and primordial monopole problems,

Phys. Lett. B 108 (1982) 389 [INSPIRE].

[7] A. Albrecht and P.J. Steinhardt, Cosmology for grand unified theories with radiatively induced symmetry breaking, Phys. Rev. Lett. 48 (1982) 1220 [INSPIRE].

[8] D.H. Lyth, What would we learn by detecting a gravitational wave signal in the cosmic microwave background anisotropy?, Phys. Rev. Lett. 78 (1997) 1861 [hep-ph/9606387] [INSPIRE].

[9] T. Li, Z. Li and D.V. Nanopoulos, Helical phase inflation, Phys. Rev. D 91 (2015) 061303 [arXiv: 1409.3267] [INSPIRE].

[10] T. Li, Z. Li and D.V. Nanopoulos, Helical phase inflation and monodromy in supergravity theory, arXiv:1412.5093 [INSPIRE].

[11] E. Cremmer, S. Ferrara, C. Kounnas and D.V. Nanopoulos, Naturally vanishing cosmological constant in $N=1$ supergravity, Phys. Lett. B 133 (1983) 61 [INSPIRE].

[12] J.R. Ellis, A.B. Lahanas, D.V. Nanopoulos and K. Tamvakis, No-scale supersymmetric standard model, Phys. Lett. B 134 (1984) 429 [INSPIRE].

[13] J.R. Ellis, C. Kounnas and D.V. Nanopoulos, Phenomenological SU $(1,1)$ supergravity, Nucl. Phys. B 241 (1984) 406 [inSPIRE].

[14] J.R. Ellis, C. Kounnas and D.V. Nanopoulos, No scale supersymmetric guts, Nucl. Phys. B 247 (1984) 373 [INSPIRE].

[15] A.B. Lahanas and D.V. Nanopoulos, The road to no scale supergravity, Phys. Rept. 145 (1987) 1 [INSPIRE].

[16] M.K. Gaillard, H. Murayama and K.A. Olive, Preserving flat directions during inflation, Phys. Lett. B 355 (1995) 71 [hep-ph/9504307] [INSPIRE]. 
[17] S. Antusch, M. Bastero-Gil, K. Dutta, S.F. King and P.M. Kostka, Solving the $\eta$-problem in hybrid inflation with Heisenberg symmetry and stabilized modulus, JCAP 01 (2009) 040 [arXiv:0808.2425] [INSPIRE].

[18] M. Kawasaki, M. Yamaguchi and T. Yanagida, Natural chaotic inflation in supergravity, Phys. Rev. Lett. 85 (2000) 3572 [hep-ph/0004243] [inSPIRE].

[19] T. Li, Z. Li and D.V. Nanopoulos, Supergravity inflation with broken shift symmetry and large tensor-to-scalar ratio, JCAP 02 (2014) 028 [arXiv:1311.6770] [INSPIRE].

[20] K. Harigaya and T.T. Yanagida, Discovery of large scale tensor mode and chaotic inflation in supergravity, Phys. Lett. B 734 (2014) 13 [arXiv: 1403.4729] [INSPIRE].

[21] T. Li, Z. Li and D.V. Nanopoulos, Symmetry breaking indication for supergravity inflation in light of the Planck 2015, JCAP 09 (2015) 006 [arXiv: 1502.05005] [INSPIRE].

[22] K. Freese, J.A. Frieman and A.V. Olinto, Natural inflation with pseudo-Nambu-Goldstone bosons, Phys. Rev. Lett. 65 (1990) 3233 [INSPIRE].

[23] F.C. Adams, J.R. Bond, K. Freese, J.A. Frieman and A.V. Olinto, Natural inflation: particle physics models, power law spectra for large scale structure and constraints from COBE, Phys. Rev. D 47 (1993) 426 [hep-ph/9207245] [INSPIRE].

[24] E. Silverstein and A. Westphal, Monodromy in the CMB: gravity waves and string inflation, Phys. Rev. D 78 (2008) 106003 [arXiv:0803.3085] [inSPIRE].

[25] L. McAllister, E. Silverstein and A. Westphal, Gravity waves and linear inflation from axion monodromy, Phys. Rev. D 82 (2010) 046003 [arXiv:0808.0706] [InSPIRE].

[26] J.E. Kim, H.P. Nilles and M. Peloso, Completing natural inflation, JCAP 01 (2005) 005 [hep-ph/0409138] [INSPIRE].

[27] K. Choi, H. Kim and S. Yun, Natural inflation with multiple sub-Planckian axions, Phys. Rev. D 90 (2014) 023545 [arXiv: 1404.6209] [INSPIRE].

[28] S.H.H. Tye and S.S.C. Wong, Helical inflation and cosmic strings, arXiv:1404.6988 [INSPIRE].

[29] R. Kappl, S. Krippendorf and H.P. Nilles, Aligned natural inflation: monodromies of two axions, Phys. Lett. B 737 (2014) 124 [arXiv:1404.7127] [INSPIRE].

[30] J. McDonald, Sub-planckian two-field inflation consistent with the Lyth bound, JCAP 09 (2014) 027 [arXiv: 1404.4620] [inSPIRE].

[31] J. McDonald, A minimal sub-planckian axion inflation model with large tensor-to-scalar ratio, JCAP 01 (2015) 018 [arXiv:1407.7471] [INSPIRE].

[32] C.D. Carone, J. Erlich, A. Sensharma and Z. Wang, Two-field axion-monodromy hybrid inflation model: Dante's waterfall, Phys. Rev. D 91 (2015) 043512 [arXiv:1410.2593] [INSPIRE].

[33] G. Barenboim and W.-I. Park, Spiral inflation, Phys. Lett. B 741 (2015) 252 [arXiv: 1412.2724] [INSPIRE].

[34] J. McDonald, Signatures of Planck corrections in a spiralling axion inflation model, JCAP 05 (2015) 014 [arXiv: 1412.6943] [INSPIRE].

[35] G. Barenboim and W.-I. Park, Spiral inflation with Coleman-Weinberg potential, Phys. Rev. D 91 (2015) 063511 [arXiv:1501.00484] [InSPIRE]. 
[36] A. Achucarro, V. Atal and Y. Welling, On the viability of $m^{2} \phi^{2}$ and natural inflation, JCAP 07 (2015) 008 [arXiv: 1503.07486] [INSPIRE].

[37] J.J. Blanco-Pillado, M. Dias, J. Frazer and K. Sousa, Large scale power suppression in a multifield landscape, arXiv:1503.07579 [INSPIRE].

[38] G. Barenboim and W.-I. Park, New- vs. chaotic-inflations, arXiv:1504.02080 [INSPIRE].

[39] M. Peloso and C. Unal, Trajectories with suppressed tensor-to-scalar ratio in aligned natural inflation, JCAP 06 (2015) 040 [arXiv: 1504.02784] [INSPIRE].

[40] Planck collaboration, P.A.R. Ade et al., Planck 2015 results. XIII. Cosmological parameters, arXiv:1502.01589 [INSPIRE].

[41] Planck collaboration, P.A.R. Ade et al., Planck 2015 results. XX. Constraints on inflation, arXiv: 1502.02114 [INSPIRE].

[42] BICEP2, Planck collaboration, P. Ade et al., Joint analysis of BICEP2/Keck Array and Planck data, Phys. Rev. Lett. 114 (2015) 101301 [arXiv:1502.00612] [INSPIRE].

[43] J. Ellis, D.V. Nanopoulos and K.A. Olive, No-scale supergravity realization of the starobinsky model of inflation, Phys. Rev. Lett. 111 (2013) 111301 [Erratum ibid. 111 (2013) 129902] [arXiv: 1305.1247] [INSPIRE].

[44] J. Ellis, D.V. Nanopoulos and K.A. Olive, Starobinsky-like inflationary models as avatars of no-scale supergravity, JCAP 10 (2013) 009 [arXiv: 1307.3537] [INSPIRE].

[45] S. Ferrara, R. Kallosh, A. Linde and M. Porrati, Minimal supergravity models of inflation, Phys. Rev. D 88 (2013) 085038 [arXiv:1307.7696] [INSPIRE].

[46] R. Kallosh, A. Linde and D. Roest, Superconformal inflationary $\alpha$-attractors, JHEP 11 (2013) 198 [arXiv: 1311.0472] [INSPIRE].

[47] J. Ellis, N.E. Mavromatos and D.V. Nanopoulos, Starobinsky-like inflation in dilaton-brane cosmology, Phys. Lett. B 732 (2014) 380 [arXiv: 1402.5075] [InSPIRE].

[48] J. Ellis, M.A.G. Garcia, D.V. Nanopoulos and K.A. Olive, A no-scale inflationary model to fit them all, JCAP 08 (2014) 044 [arXiv: 1405.0271] [INSPIRE].

[49] C. Kounnas, D. Lüst and N. Toumbas, $R^{2}$ inflation from scale invariant supergravity and anomaly free superstrings with fluxes, Fortsch. Phys. 63 (2015) 12 [arXiv:1409.7076] [INSPIRE].

[50] J. Ellis, M.A.G. Garcia, D.V. Nanopoulos and K.A. Olive, Two-field analysis of no-scale supergravity inflation, JCAP 01 (2015) 010 [arXiv:1409.8197] [INSPIRE].

[51] T. Higaki and F. Takahashi, Elliptic inflation: interpolating from natural inflation to $R^{2}$-inflation, JHEP 03 (2015) 129 [arXiv:1501.02354] [INSPIRE].

[52] K. Kannike et al., Dynamically induced Planck scale and inflation, JHEP 05 (2015) 065 [arXiv: 1502.01334] [INSPIRE].

[53] M. Ozkan, Y. Pang and S. Tsujikawa, Planck constraints on inflation in auxiliary vector modified $f(R)$ theories, Phys. Rev. D 92 (2015) 023530 [arXiv:1502.06341] [INSPIRE].

[54] J. Ellis, M.A.G. Garcia, D.V. Nanopoulos and K.A. Olive, No-scale inflation, arXiv: 1507.02308 [INSPIRE].

[55] M. Cicoli, C.P. Burgess and F. Quevedo, Fibre inflation: observable gravity waves from IIB string compactifications, JCAP 03 (2009) 013 [arXiv: 0808.0691] [INSPIRE]. 
[56] C.P. Burgess, M. Cicoli and F. Quevedo, String inflation after Planck 2013, JCAP 11 (2013) 003 [arXiv: 1306.3512] [INSPIRE].

[57] M. Cicoli, S. Downes and B. Dutta, Power suppression at large scales in string inflation, JCAP 12 (2013) 007 [arXiv: 1309.3412] [INSPIRE].

[58] R. Blumenhagen, A. Font, M. Fuchs, D. Herschmann and E. Plauschinn, Towards axionic Starobinsky-like inflation in string theory, Phys. Lett. B 746 (2015) 217 [arXiv:1503.01607] [INSPIRE].

[59] S. Kachru, M.B. Schulz, P.K. Tripathy and S.P. Trivedi, New supersymmetric string compactifications, JHEP 03 (2003) 061 [hep-th/0211182] [INSPIRE].

[60] J. Shelton, W. Taylor and B. Wecht, Nongeometric flux compactifications, JHEP 10 (2005) 085 [hep-th/0508133] [INSPIRE].

[61] G. Aldazabal, P.G. Camara, A. Font and L.E. Ibáñez, More dual fluxes and moduli fixing, JHEP 05 (2006) 070 [hep-th/0602089] [INSPIRE].

[62] J. Shelton, W. Taylor and B. Wecht, Generalized flux vacua, JHEP 02 (2007) 095 [hep-th/0607015] [INSPIRE].

[63] A. Micu, E. Palti and G. Tasinato, Towards Minkowski vacua in type II string compactifications, JHEP 03 (2007) 104 [hep-th/0701173] [INSPIRE].

[64] A. Font, A. Guarino and J.M. Moreno, Algebras and non-geometric flux vacua, JHEP 12 (2008) 050 [arXiv:0809.3748] [INSPIRE].

[65] A. Guarino and G.J. Weatherill, Non-geometric flux vacua, S-duality and algebraic geometry, JHEP 02 (2009) 042 [arXiv: 0811.2190] [INSPIRE].

[66] B. de Carlos, A. Guarino and J.M. Moreno, Complete classification of Minkowski vacua in generalised flux models, JHEP 02 (2010) 076 [arXiv:0911.2876] [INSPIRE].

[67] G. Aldazabal, D. Marques, C. Núñez and J.A. Rosabal, On Type IIB moduli stabilization and $N=4,8$ supergravities, Nucl. Phys. B 849 (2011) 80 [arXiv:1101.5954] [INSPIRE].

[68] F. Hassler, D. Lüst and S. Massai, On inflation and de Sitter in non-geometric string backgrounds, arXiv:1405.2325 [INSPIRE].

[69] R. Blumenhagen et al., A flux-scaling scenario for high-scale moduli stabilization in string theory, Nucl. Phys. B 897 (2015) 500 [arXiv:1503.07634] [inSPIRE].

[70] S.V. Ketov and T. Terada, Single-superfield helical-phase inflation, arXiv:1509.00953 [INSPIRE].

[71] D. Andriot and A. Betz, Supersymmetry with non-geometric fluxes, or a $\beta$-twist in generalized geometry and Dirac operator, JHEP 04 (2015) 006 [arXiv:1411.6640] [INSPIRE].

[72] L.E. Ibanez and A.M. Uranga, String theory and particle physics: an introduction to string phenomenology, Cambridge University Press, Cambridge U.K. (2012.

[73] S. Gukov, C. Vafa and E. Witten, CFT's from Calabi-Yau four folds, Nucl. Phys. B 584 (2000) 69 [Erratum ibid. B 608 (2001) 477] [hep-th/9906070] [INSPIRE].

[74] D. Robbins and T. Wrase, D-terms from generalized NS-NS fluxes in type-II, JHEP 12 (2007) 058 [arXiv:0709.2186] [INSPIRE].

[75] P. Shukla, On modular completion of generalized flux orbits, arXiv:1505.00544 [INSPIRE]. 
[76] P. Shukla, Implementing odd-axions in dimensional oxidation of non-geometric type IIB action, arXiv: 1507.01612 [INSPIRE].

[77] J. Gray, Y.-H. He, A. Ilderton and A. Lukas, STRINGVACUA: a Mathematica package for studying vacuum configurations in string phenomenology,

Comput. Phys. Commun. 180 (2009) 107 [arXiv:0801.1508] [InSPIRE].

[78] S. Kachru, M.B. Schulz and S. Trivedi, Moduli stabilization from fluxes in a simple IIB orientifold, JHEP 10 (2003) 007 [hep-th/0201028] [INSPIRE].

[79] O. DeWolfe, A. Giryavets, S. Kachru and W. Taylor, Enumerating flux vacua with enhanced symmetries, JHEP 02 (2005) 037 [hep-th/0411061] [INSPIRE]. 